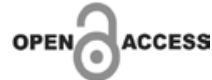

Correspondence addresses: Dr. Gilson Soares Feitosa gilson-feitosa@uol.com.br

Received: April 8, 2021

Revised: May 21, 2021

Accepted: June 14, 2021

Published: June 30, 2021

Data Availability Statement: All relevant data are within the paper and its Supporting Information files.

Funding: This work was the result of authors' initiative. There was no support of research or publication funds.

Competing interests: The authors have declared that no competing interests exist.

Copyright

(C) 2021 by Santa Casa

de Misericórdia da Bahia.

All rights reserved.

ISSN: $2526-5563$

DOI: 10.35753
ATUALIZAÇÃO DE TEMA

\section{Desafios na Transposição dos Conhecimentos em Hipertensão Arterial para a Prática Médica}

\author{
The Challenges in Conveying Hypertension Knowledge to \\ Clinical Practice
}

\author{
Gilson Feitosa ${ }^{12^{*}}$, Isabela Pilar ${ }^{2}$, Victor F. Seabra ${ }^{3}$ \\ ${ }^{1}$ Escola Bahiana de Medicina e Saude Pública: ${ }^{2}$ Serviço de Cardiologia do Hospital Santa \\ Izabel da Santa Casa da Bahia; Salvador, Bahia: ${ }^{3}$ Hospital das Clínicas da Faculdade de \\ Medicina da Universidade de São Paulo; São Paulo, São Paulo, Brazil
}

\begin{abstract}
A despeito do volume de conhecimentos adquiridos sobre a hipertensão arterial com a descoberta de seus determinantes e desenvolvimento de intervenções preventivas e terapêuticas eficazes, permanece ainda um grande desafio transportar para a prática diária tais conhecimentos. A prevenção da hipertensão arterial depende de intensa atividade educacional dirigida à população. Os recursos farmacológicos permitem o controle adequado da imensa maioria dos casos. No entanto, casos de difícil controle são consequência de uma ampla gama de possibilidades de ordem comportamental e estrutural. $E$ os casos verdadeiramente resistentes poderão ser objeto de recursos auxiliares com dispositivos com atenção voltada para a desnervação renal.

Palavras-chave: Hipertensão Arterial; Hipertensão Arterial Resistente;Hipertensão Arterial Refratária; Tratamento Farmacológico; Desnervação Renal.
\end{abstract}

The adequate control of blood pressure is still a challenge, despite a large amount of knowledge about hypertension, the determinants and the development of preventive resources, and the efficacy of pharmacologic treatment. Educational programs directed to the general population are essential for hypertension prevention. Pharmacologic resources are capable of achieving adequate control on the majority of cases. However, behavioral and structural problems result in cases of difficulty management. And Truly resistant cases may be the object of interventional therapies, especially those involving renal denervation procedures.

Keywords: High Blood Pressure; Resistant Hypertension; Refractory Hypertension; Pharmacologic Treatment; Renal Denervation.

\section{Introdução}

A despeito dos avanços do conhecimento em hipertensão arterial desde o final do século XVIII, com as experiências cruentas de medidas da pressão arterial de forma direta, passaram-se mais de 100 anos para que se desenvolvessem recursos para aferição de forma indireta da medida da pressão arterial por método auscultatório, ou oscilométrico. ${ }^{1} \mathrm{E}$, quando foi possível aferir esta variável de forma disseminada na prática clínica, muito se acumulou de conhecimento em torno desta entidade nosológica nos últimos anos e, principalmente, a partir de meados do século 20. 
Foi então observado que indivíduos que mantivessem cifras pressóricas acima de 140 por $90 \mathrm{mmHg}$ apresentavam risco elevado ao longo da vida para o desenvolvimento de manifestações cardiovasculares tais como: infarto agudo do miocárdio, insuficiência cardíaca, acidente vascular cerebral entre outras patologias.

Por exemplo, sabe-se que a hipertensão arterial está presente na história de metade dos pacientes que sofrem infarto agudo do miocárdio e insuficiência cardíaca, e cerca de $70 \%$ dos pacientes que têm acidente vascular cerebral, o que denota a sua importância para a Medicina e saúde púbica. ${ }^{2}$

Como desde meados do século 20 passouse a reconhecer as doenças cardiovasculares como a principal causa de morte, houve uma intensa concentração de interesse em se conhecer mecanismos de determinação da hipertensão arterial e, principalmente, em executar adequadamente sua prevenção e seu tratamento. Desde então, acumulam-se os conhecimentos em torno desta entidade nosológica em várias partes do mundo sob a forma de livros e periódicos, com milhares de artigos publicados a respeito.

Afora isso, a prevalência da hipertensão arterial é reconhecidamente muito elevada nas distintas populações do mundo. Na população adulta brasileira, ${ }^{3}$ observa-se uma prevalência que é crescente com o aumento da faixa etária, atingindo proporções muito significativas em indivíduos acima dos 50 a 60 anos, podendo ser observada em até 40 a 50\% dos indivíduos além dessa idade (Figura 1).

Desta forma, o aumento da hipertensão arterial com o avanço da idade é um dos principais motivos para o interesse desenvolvido pelas entidades científicas e sanitárias observada em distintas partes do mundo.

Esse aumento progressivo da prevalência da hipertensão arterial com o crescimento da faixa etária dá-nos uma ideia clara de que a hipertensão tem uma incidência crescente com o passar da idade, o que é comprovado com uma interessante observação feita no estudo Framingham (Quadro 1), ${ }^{4}$ em que indivíduos entre os 35 e 64 anos de

Figura 1. Prevalência da hipertensão arterial na população brasileira.

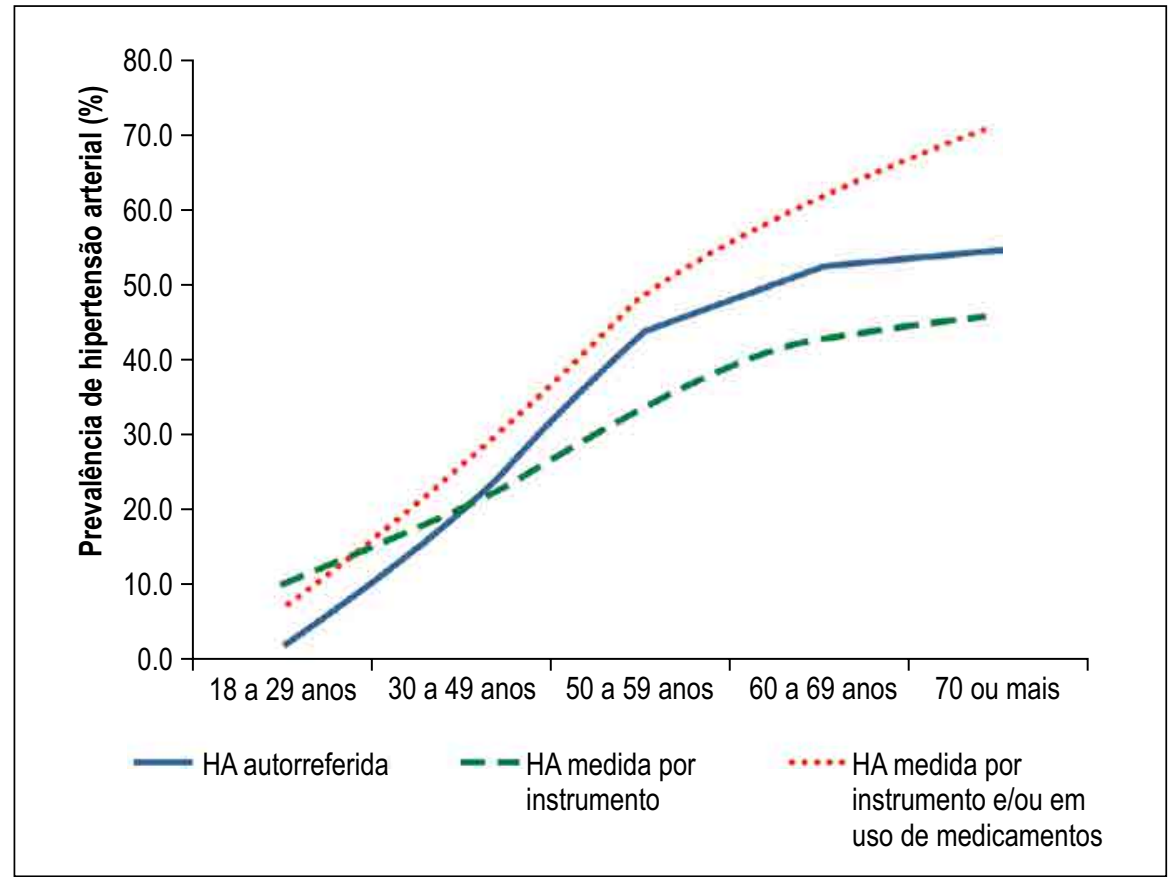

HA autorreferida $=$ conforme o conhecimento do paciente. HA medida por instrumento $=\mathrm{PA}>140 / 90 \mathrm{mmHg}$. HA medida por instrumento e/ou em uso de medicamentos $=$ PA $>140 / 90$ mmHg, ou PA $\leq 140 / 90$ mmHg com uso de medicação anti-hipertensiva. 
idade, vistos 4 anos depois de uma observação inicial, têm uma chance de desenvolvimento de hipertensão de 5,3\%, 17,6\%, ou $37,3 \%$ se apresentavam inicialmente uma cifra pressórica que era considerada ótima (abaixo de 120/80 $\mathrm{mmHg}$ ), normal (de 121 a $129 \mathrm{mmHg}$ para a sistólica ou de 80 a $84 \mathrm{mmHg}$ para a diastólica), ou pré-hipertensão (entre 130 e $139 \mathrm{mmHg}$ para a sistólica e 85 a $89 \mathrm{mmHg}$ para a diastólica), respectivamente. Sendo que esses números eram ainda mais elevados, da ordem de $16 \%$, $25,6 \%$ e $49,5 \%$, para aqueles indivíduos que inicialmente tivessem sido vistos com a idade entre 65 e 94 anos. Isto dá uma ideia muito clara da perspectiva de desenvolvimento de hipertensão arterial, principalmente naqueles indivíduos que se apresentam com cifras consideradas como de pré-hipertensão.

\section{O Primeiro Desafio: APrevenção da Hipertensão Arterial}

Dessa forma, delineia-se o primeiro grande desafio, qual seja, o de prevenir o surgimento de hipertensão arterial.
Medidas comportamentais e dietéticas podem desempenhar um importante papel nesse contexto. ${ }^{5}$ Por exemplo, o controle do peso está entre as medidas mais efetivas para este propósito havendo uma estimativa de que, em indivíduos com sobrepeso, possa haver redução de $1 \mathrm{mmHg}$ de pressão sistólica para cada quilograma de peso reduzido. Já na prevenção primária da hipertensão arterial esse controle de peso, na prática, associase a uma redução em torno de 2 a $3 \mathrm{mmHg}$ da pressão arterial sistólica.

A adoção de uma dieta saudável, rica em frutas, vegetais e grãos, e com baixo teor de gordura, principalmente com redução de gordura saturada e redução de ácidos graxos trans, tem a perspectiva de, em prevenção primária, diminuir a pressão arterial em cerca de $3 \mathrm{mmHg}$.

$\mathrm{O}$ estabelecimento de uma dieta hipossódica, considerando-se uma redução da ingestão de sódio aquém de $2 \mathrm{~g}$ por dia, associa-se a uma redução de pressão arterial sistólica de 2 a $3 \mathrm{mmHg}$.

Por outro lado, um aumento da ingestão de potássio na dieta, da ordem de 3 a 5 gramas por dia, reduz a pressão arterial em prevenção primária em torno de $2 \mathrm{mmHg}$.

Quadro 1. Incidência de hipertensão arterial em 4 anos.

Categoria da PA Basal

\begin{tabular}{|l|c|c|}
\hline \multirow{2}{*}{$\begin{array}{l}\text { Ótima } \\
(\mathrm{PAS}<120 \mathrm{mmHg} \text { e PAD }<80 \\
\mathrm{mmHg})\end{array}$} & Idade 35-64 anos & Idade 65 - 94 anos \\
\cline { 2 - 3 } & $5,3(4,4-6,3)$ & $16,0(12,0-20,9)$ \\
$\begin{array}{l}\text { Normal } \\
\text { (PAS de } 120 \text { a } 129 \mathrm{mmHg} \text { e } \\
\text { PAD de } 80 \text { a } 84 \mathrm{mmHg})\end{array}$ & $17,6(17,2-20,3)$ & $25,5(20,4-31,4)$ \\
$\begin{array}{l}\text { Pré-hipertensão } \\
\text { PAS de } 130 \text { a } 139 \mathrm{mmHg} \text { e }\end{array}$ & $37,3(33,3-41,5)$ & $49,5(42,6-56,4)$ \\
PAD 85 a $89 \mathrm{mmHg}$ & & \\
\hline
\end{tabular}

(IC= intervalo de confiança).* Taxas são por 100, e foram ajustados por sexo, idade, índice de massa corpórea; PA sistólica e diastólica no basal. 
A atividade física é vista como importante para auxiliar na prevenção primária de hipertensão arterial, contribuindo para isso formas de exercício de resistência dinâmica assim como de atividade aeróbica. Quando convenientemente executados, estima-se que exercícios físicos reduzam a pressão em 5 a 7 mmHg. ${ }^{6}$

O consumo de álcool também reduz a pressão arterial desde que feito de maneira moderada, o que significa menos de 2 drinques por dia para homens, e menos de um drinque por dia para mulheres. Consumido dessa maneira, associa-se a uma redução de até 4 a $5 \mathrm{mmHg}$.

Diante de todo esse conhecimento qual tem sido o resultado da aplicação dessas medidas não farmacológicas na prática?

Tal conhecimento não tem sido absorvido adequadamente pelas populações (Figura 2) ${ }^{6}$
Por exemplo, comparando-se dois períodos de observação: o período do ano 2000 e o período do ano 2010, verificou-se um aumento na prevalência de hipertensão arterial na população na maioria das regiões do mundo, principalmente nos países menos desenvolvidos. Em países de alto poder econômico e consequentemente nível educacional mais elevado, registou-se um leve decréscimo da prevalência de hipertensão arterial, tanto para homens como para mulheres, da ordem de 3,6 e 1,7\%, respectivamente. $\mathrm{Na}$ maioria das demais regiões do mundo, o que se observou foi um aumento da prevalência de hipertensão arterial. O que nos leva a querer entender em sua essência quais são verdadeiramente os determinantes da hipertensão arterial sistêmica.

Sabe-se que os fatores determinantes de hipertensão $\operatorname{arterial}^{7}$ podem ser vistos de três naturezas distintas:

Figura 2. Prevalência de hipertensão arterial em diferentes regiões do mundo em 2 períodos distintos: 2000 e 2010.

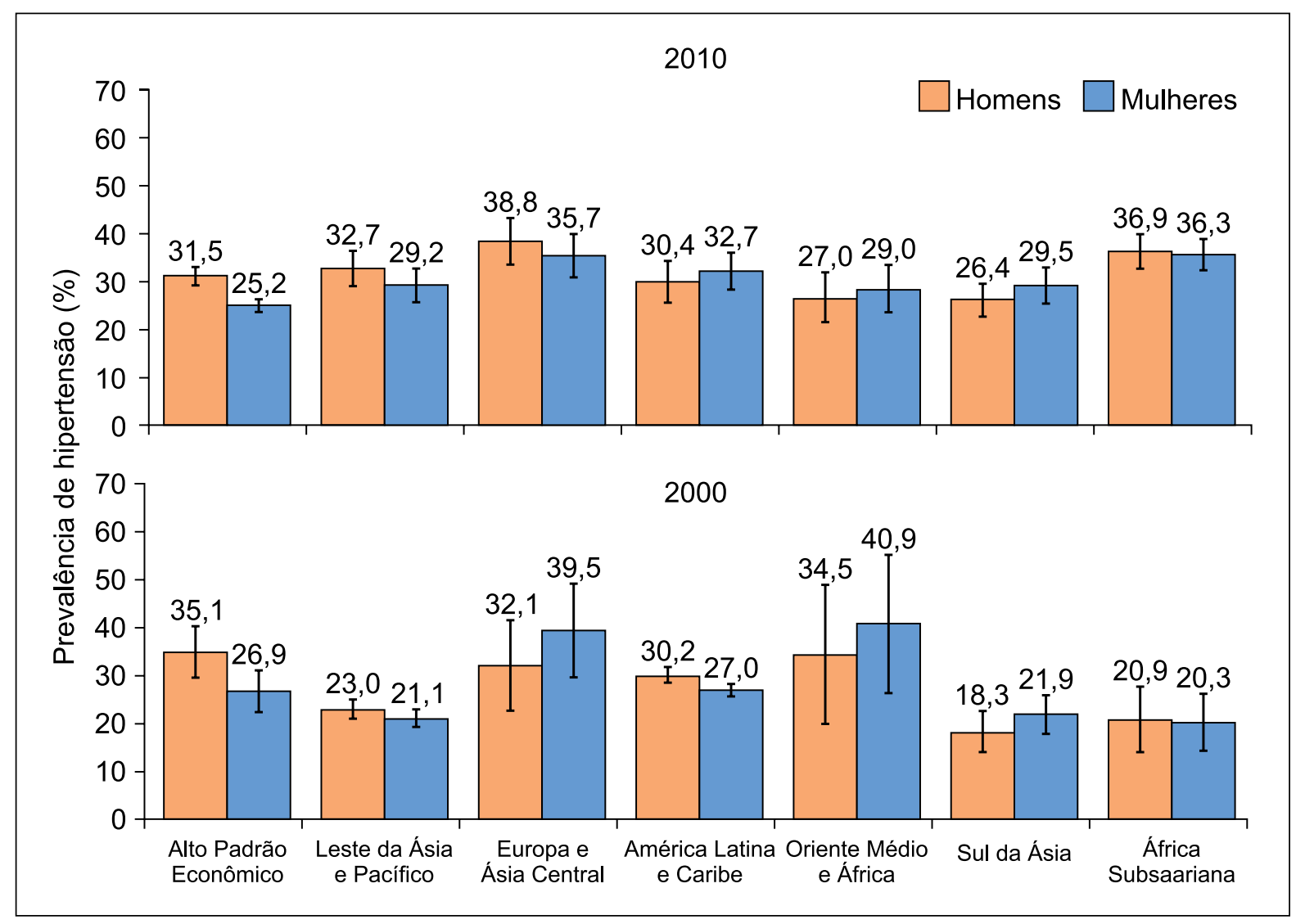


a) Os determinantes sociais;

b) Os determinantes ambientais;

c) Os determinantes genéticos e epigenéticos

Os determinantes genéticos e epigenéticos têm sido motivo de muitos estudos que levam à conclusão de que são múltiplos os alelos que interagem de modo a determinar a hipertensão, mas, individualmente, cada um tem pequena força de expressão.

Ultimamente, presta-se muita atenção a fenômenos epigenéticos que modificam a constituição genética inicial do indivíduo, sob a influência de fatores ambientais. Defeitos monogênicos que levam à hipertensão arterial na realidade são raros.

Entende-se que a interação entre fatores ambientais relacionados ao sedentarismo, uma dieta com excesso de sal, o aparecimento de obesidade e fatores sociais, como baixo nível de educação, dificuldade de acesso a sistemas de saúde, além de pobreza, são determinantes mais importantes, ensejando essa prevalência tão alta de hipertensão arterial no mundo, principalmente nos países pobres.

Até esse momento, as estratégias desenvolvidas para prevenir a hipertensão arterial encontram-se sob duas vertentes: a mais utilizada é a de uma atenção individual em que se procura identificar os indivíduos de maior risco, que são os pré-hipertensos, e neles concentrar esforços no sentido de educação, de modo a implementar a correção de desvios de fatores que levam a esta condição. Uma outra vertente é a de dirigir os esforços para o controle em âmbito populacional. Sabe se que esta é a mais efetiva.Por exemplo, se houver na população em geral uma redução da pressão arterial diastólica de $2 \mathrm{mmHg}$, estimase que isso está associada a uma diminuição de $17 \%$ na incidência de hipertensão arterial sistêmica. ${ }^{8}$ Ocorre que as medidas gerais dirigidas à população têm sido difíceis de serem implementadas, mas certamente são um alvo de interesse da saúde pública.

\section{O Segundo Desafio: O Controle da Pressão Arterial no Paciente Hipertenso}

O outro grande desafio no campo da hipertensão arterial é o de garantir o seu controle uma vez que a hipertensão arterial já esteja presente.

Nesse sentido, grande tem sido o investimento na identificação de recursos farmacológicos que ajudem na redução e controle da pressão arterial. $\mathrm{O}$ que tem sido alcançado com muito sucesso.

Uma intensa procura desses recursos farmacológicos que procura aliar eficácia antihipertensiva a uma tolerabilidade aceitável inicia-se desde os anos 50 do século passado e progressivamente um grande número de medicamentos foram surgindo que podem fundamentalmente ser identificados como pertencentes a quatro ou cinco grupos: os diuréticos, destacando-se aqui os tiazídicos, os beta-bloqueadores, os antagonistas de canais de cálcio, os atenuadores do sistema renina angiotensina, a saber, os inibidores da enzima de conversão da angiotensina e os antagonistas dos receptores tipo 1 da angiotensina 2 (Quadro 2).

Esses medicamentos utilizados isoladamente, ou em combinação, são capazes de controlar a imensa maioria de casos de hipertensão arterial, atingindo as metas pressóricas que sejam estabelecidas.

Eles são amplamente disponíveis e geralmente bem tolerados.

Dessa forma é surpreendente o que se observa de real controle de hipertensão arterial para as metas pré-estabelecidas na população de hipertensos.

Em ensaios clínicos, em que há todo o suporte de cuidados oferecidos aos pacientes, ainda assim verifica-se a falta de sucesso no controle de metas pressóricas, variando de 18 a 55\% (Figura 3).

No Serviço do Hospital Santa Isabel (Salvador, Bahia, Brasil), realizou-se um projeto de verificação do uso do pedômetro no auxílio ao estímulo da atividade física entre pacientes hipertensos. ${ }^{9}$ Entre outras demandas, tal projeto 
Quadro 2. Evolução farmacológica no tratamento da hipertensão arterial.

\begin{tabular}{|c|c|c|c|c|c|}
\hline \multicolumn{6}{|c|}{ TRATAMENTO DE H.A.S. } \\
\hline $1940-50$ & $1950-60$ & $1960-70$ & $1970-80$ & $1980-90$ & $1990-\ldots$ \\
\hline DIETA RED. NA & DIETA RED. NA & DIETA RED. NA & DIETA RED. NA & DIETA RED. NA & \\
\hline \multirow[t]{14}{*}{$\begin{array}{l}\text { SIMPATECTOMIA } \\
\text { CIRÚRGICA }\end{array}$} & $\begin{array}{l}\text { SIMPATECTOMIA } \\
\text { CIRÚRGICA }\end{array}$ & $X X X X X X$ & $X X X X X X$ & $X X X X X X$ & \\
\hline & $\begin{array}{l}\text { BLOQUEADORES } \\
\text { GANGLIONARES }\end{array}$ & $\begin{array}{l}\text { BLOQUEADORES } \\
\text { GANGLIONARES }\end{array}$ & $X X X X X X$ & $X X X X X X$ & \\
\hline & RESERPINA & RESERPINA & RESERPINA & $X X X X X X$ & \\
\hline & HIDRALAZINA & HIDRALAZINA & HIDRALAZINA & HIDRALAZINA & \\
\hline & GUANETIDINA & GUANETIDINA & GUANETIDINA & GUANETIDINA & \\
\hline & TIAZÍDICOS & TIAZÍDICOS & TIAZÍDICOS & TIAZÍDICOS & \\
\hline & & ESFIRONOLACTONA & ESFIRONOLACTONA & ESFIRONOLACTONA & \\
\hline & & ALFAMETILDOPA & ALFAMETILDOPA & ALFAMETILDOPA & \\
\hline & & FUROSEMIDA & FUROSEMIDA & FUROSEMIDA & \\
\hline & & & DIAZOXIDE & DIAZOXIDE & \\
\hline & & & MINOXIDIL & MINOXIDIL & \\
\hline & & & PRAZOSIN & PRAZOSIN & \\
\hline & & & BETABLOQUEADOR & BETABLOQUEADOR & \\
\hline & & & & $\frac{\text { ANTAG. CA }}{\text { I. ECA. }}$ & AAll \\
\hline
\end{tabular}

Antag. $\mathrm{Ca}=$ antagonistas de canais de cálcio; $\mathrm{I} . \mathrm{ECA}=$ inibidores da enzima de conversão da angiotensina; $\mathrm{AAII}=$ antagonistas dos receptores da angiotensina II (ou $\mathrm{BRA}=$ bloqueador do receptor da angiotensina II).

serviu para mostrar que mesmo no ambulatório especializado de hipertensão arterial, $25 \%$ dos pacientes não atingiram o adequado controle, rotulados, pois, como pacientes de difícil controle da hipertensão arterial.

\section{Que Razões Existiriam para Tal?}

Os anti-hipertensivos são muito semelhantes no que diz respeito à sua eficácia anti-hipertensiva, à exceção dos beta-bloqueadores que, em relação aos demais, têm comprovadamente uma eficácia menor.

Houve por um tempo a observação de que os diuréticos seriam capazes de induzir o surgimento subsequente de diabetes mellitus. A rigor, não se confirma que isso seja necessariamente relevante e, na realidade, os benefícios alcançados com o emprego do anti-hipertensivo depende muito mais do grau de controle atingido do que com o seu tipo farmacológico específico. ${ }^{10} \mathrm{O}$ que de fato os distingue de alguma forma é uma melhor tolerabilidade ao uso dos inibidores de ECA e os BRAs, notadamente estes últimos. ${ }^{11}$ Isto tem feito com que no processo inicial de escolha de um antihipertensivo eles venham ganhando maior espaço.

Há, no entanto, em algumas partes do mundo as limitações relacionadas ao custo dos medicamentos, sendo aqui os diuréticos os medicamentos mais acessíveis.

Mas, a rigor, suas diferenças em termos de eficácia e benefícios são muito pequenas, de modo que o melhor anti-hipertensivo é, como diria o hipertensólogo Décio Mion, aquele que o paciente possa usar.

Em pacientes que não atingem a meta de controle pressórico existem várias razões possíveis para tal dificuldade. Incluem-se entre elas: pseudo resistência ao tratamento, a falta de aderência às medicações prescritas assim como a falta de 
Figura 3. Sucesso de alcance da meta pressórica em ensaios clínicos.

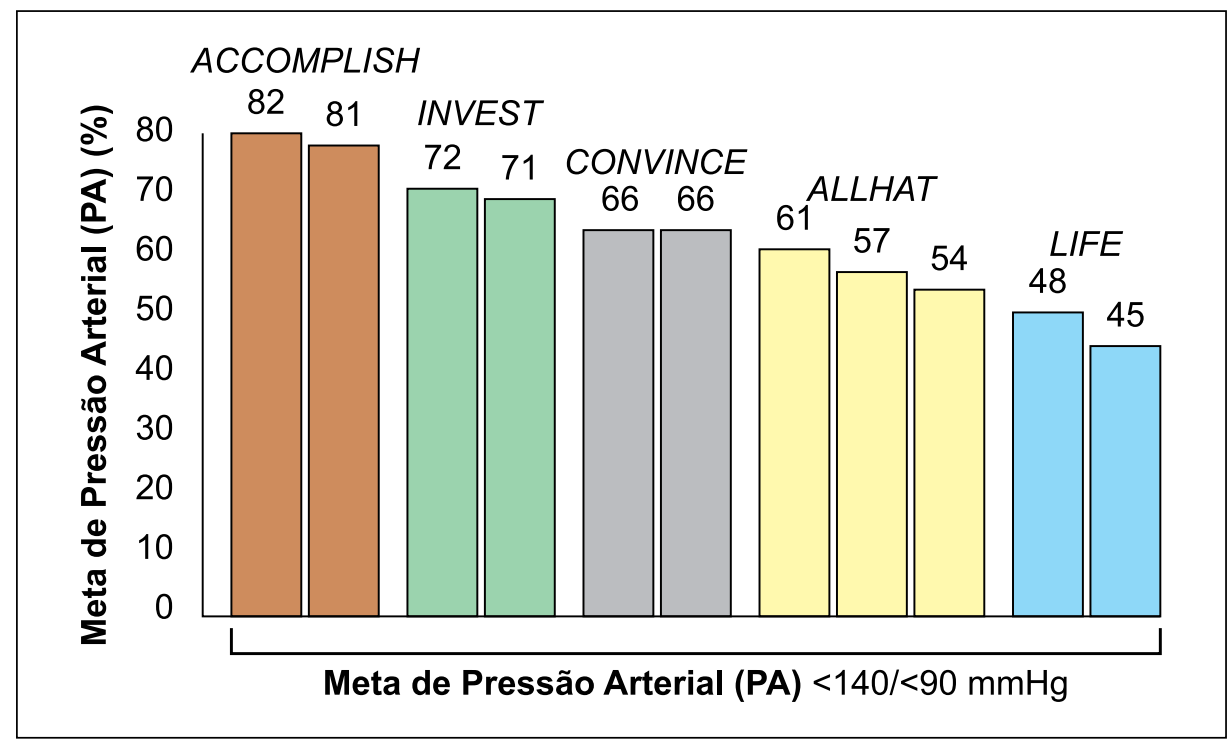

Ensaios Clínicos: ACCOMPLISH (Avoiding Cardiovascular Events Through Combination Therapy in Patients Living With Systolic Hypertension / Evitando Eventos Cardiovasculares por Meio de Terapia Combinada em Pacientes com Hipertensão Sistólica): amlodipine + benazepril versus benazepril + hidroclorotiazida; INVEST (International Verapamil-Trandolapril Study / Estudo Internacional VerapamilTrandolapril): verapamil versus atenolol; CONVINCE (Controlled Onset Verapamil Investigation of Cardiovascular End Points / Investigação Inicial Controlada com Verapamil sobre Desfechos Finais Cardiovasculares): verapamil versus atenolol (57); ALLHAT (Antihypertensive and Lipid-Lowering Treatment to Prevent Heart Attack Trial / Ensaio Clínico de Tratamento Anti-Hipertensivo e Hipolipemiante para Prevenir Ataques Cardíacos): clortalidona versus lisinopril versus amlodipine; and LIFE (Losartan Intervention For Endpoint Reduction in Hypertension Study / Participação da Losartana para Redução de Desfechos Finais em Estudo de Hipertensão): losartana versus atenolol.

aderência à dieta recomendada. Mas, em especial, ressalta-se a inércia do médico em ajustar as doses do medicamento.

Embora isto pareça muito simples, em um estudo realizado em cinco países europeus como França, Alemanha, Itália, Espanha e Reino Unido, verificou-se que diante da constatação da cifra pressórica acima da meta em 7.246 pacientes hipertensos, os médicos mantiveram a prescrição em $84 \%$ dos casos. Esse aspecto de educação médica é, portanto, de interesse no tratamento de hipertensos.

Diante de casos de hipertensão de difícil controle há de se considerar formas resistentes de hipertensão cuja definição é: "Manutenção das cifras pressóricas acima das metas de controle mesmo que em uso adequado de medicações de 3 classes distintas de anti-hipertensivos, idealmente incluindo um diurético." 12

Em casos assim há de se insistir sempre em excluir uma pseudo resistência por conta de transgressões dietéticas ou do uso dos medicamentos, além de se procurar outros fatores que concorram para esta resistência como os ligados a idade mais avançada, obesidade, síndrome da apneia obstrutiva do sono, entre outros. Há de se considerar o uso de outras medicações concomitantes e que possam dificultar o controle da pressão arterial como por exemplo o uso de descongestionantes nasais, corticosteroides e outras medicações utilizadas em condições específicas, como ciclosporina, eritropoietina, 
entre outras. No grupo de pacientes definidos como portadores de hipertensão arterial resistente, a prevalência de formas secundárias de hipertensão é maior e estas devem ser procuradas.

Em estudo realizado com o propósito de definir qual é realmente a incidência de casos de hipertensão arterial existente entre novos casos de hipertensos, chegou-se à conclusão de que a hipertensão arterial verdadeiramente resistente encontra-se em torno de $2 \%$ dos casos quando todas essas outras condições abordáveis são excluídas. ${ }^{13} \mathrm{Já}$ em um estudo populacional brasileiro sua prevalência foi de $11 \%{ }^{14}$

Ainda assim, reconhece-se sua importância posto que tais pacientes reservam um pior prognóstico sendo demonstrada uma incidência de eventos cardiovasculares nestes pacientes de cerca de $48 \%$ dos casos em 3,8 anos. $^{13}$

\section{A Quarta Droga no Tratamento da Hipertensão Resistente}

Em pacientes com hipertensão arterial resistente, duas medicações têm merecido destaque especial, a saber: a espironolactona e a clonidina.

Em estudo realizado no Brasil ${ }^{14}$ com participação do Serviço de Hipertensão Arterial do Hospital Santa Isabel (estudo REHOT), foi possível fazer uma comparação entre essas duas medicações, demonstrando-se que ambas auxiliam no controle da pressão arterial de casos resistentes, sendo, no entanto, a espironolactona mais efetiva.

Vários outros estudos corroboram essa observação e tem-se bem assentado então que a espironolactona deva ser a quarta droga no esquema de tratamento.

Eventualmente, há necessidade do acréscimo de uma quinta ou até mais drogas.

\section{Recursos Não-Farmacológicos para o Trata- mento da Hipertensão Arterial Refratária}

No paciente que não se obtém o controle da cifra pressórica com cinco ou mais drogas distintas, tem-se o diagnóstico de hipertensão arterial refratária.

Para esse tipo de paciente há uma busca intensa de novos recursos que ajudem na obtenção do controle dessa hipertensão arterial e que são ainda considerados como estudos experimentais.

Dentre esses destacam-se a estimulação do seio carotídeo, a desnervação renal, o uso de fistulas arteriovenosas, entre outros.

De todos esses, o método que está mais avançado é o da desnervação renal.

$\mathrm{O}$ interesse em torno dessa estratégia já existe há algum tempo consistindo basicamente da tentativa de promover uma desnervação da artéria renal por meio de um processo endovascular ablativo obtido por diferentes métodos tais como por radiofrequência, por meio de um cateterismo seletivo de artérias renais. Estabeleceu-se uma série de estudos dentro de um programa que foi chamado de Simplicity ${ }^{15}$ e que demonstrou que a ablação por rádiofrequência era capaz de reduzir tanto a pressão arterial sistólica como a pressão arterial diastólica de pacientes com hipertensão arterial resistente. À medida em que o grau de exigência da comprovação de sua eficácia foi crescendo, tornou-se necessária que esta experimentação fosse comparada a um procedimento sham. Isto é, um procedimento real foi comparado de maneira randomizada a procedimento simulado com o desconhecimento da natureza do mesmo por parte do paciente.

Este estudo chamou-se Simplicity $3^{16}$ e, surpreendentemente, revelou que os resultados de redução da pressão arterial observados ao longo do tempo foram semelhantes no grupo que fez o procedimento e no grupo que fez o simulacro do procedimento, pondo dúvidas sobre a real aplicação desse método. Isso interrompeu temporariamente o grande entusiasmo que se iniciava em torno dessa técnica inclusive com a apresentação de dezenas de sistemas já sendo estudados para promover tal ablação.

Uma análise crítica do que foi feito, ${ }^{17}$ inclusive no estudo Simplicity 3, deixa dúvidas quanto à técnica de obtenção do resultado proposto, de 
modo que outros investigadores se puseram em campo explorando outras opções. Foram então apresentados recentemente resultados com o sistema de desnervação renal a base de ultrassom.

O mais recente desses estudos foi o estudo intitulado Radiance - HTN Trio ${ }^{18}$ que partiu do princípio da termogênese gerada pelo recurso ultrassônico, e que em modelos experimentais parece proporcionar uma desnervação mais consistente. Este foi um estudo muito cuidadoso também controlado por um grupo sham. Houve o cuidado de uma verificação da aderência às medicações em ambos os grupos, pela medida de excreção urinária das medicações que estiveram em uso (Figura 4).

Dessa forma, confirmou-se uma eficácia de redução em dois meses da pressão arterial sistólica e diastólica como vistas pela monitorização ambulatorial de pressão arterial.

A isto somaram-se outras evidências recentes a partir de uma metodologia semelhante porém com novos catéteres em espiral como parte dos programas Spiral Hypertension, ${ }^{19}$ mostrando eficácia quando comparada a um procedimento sham.

Dessa forma, restabelece-se então o interesse continuado no desenvolvimento futuro próximo de técnicas auxiliares não-farmacológicas, aplicáveis de início para aqueles pacientes com hipertensão arterial refratária.

\section{Conclusão}

Há uma considerável dificuldade de implementação real de recursos já disponíveis e que comprovadamente levam a um controle mais eficaz da pressão arterial na população em geral e no indivíduo hipertenso.

Do ponto de vista populacional, ações informativas promovidas pelas autoridades sanitárias e sociedades científicas constituem-se por certo na medida esperada a contribuir para uma redução mais abrangente, lembrando que fatores sociais desempenham um importante papel na determinação de hipertensão arterial tais como educação e acesso a serviços de saúde.

Figura 4. Resultado do estudo RADIANCE-HTN Trio.

\section{ABPM profiles at Baseline and 2 Months}

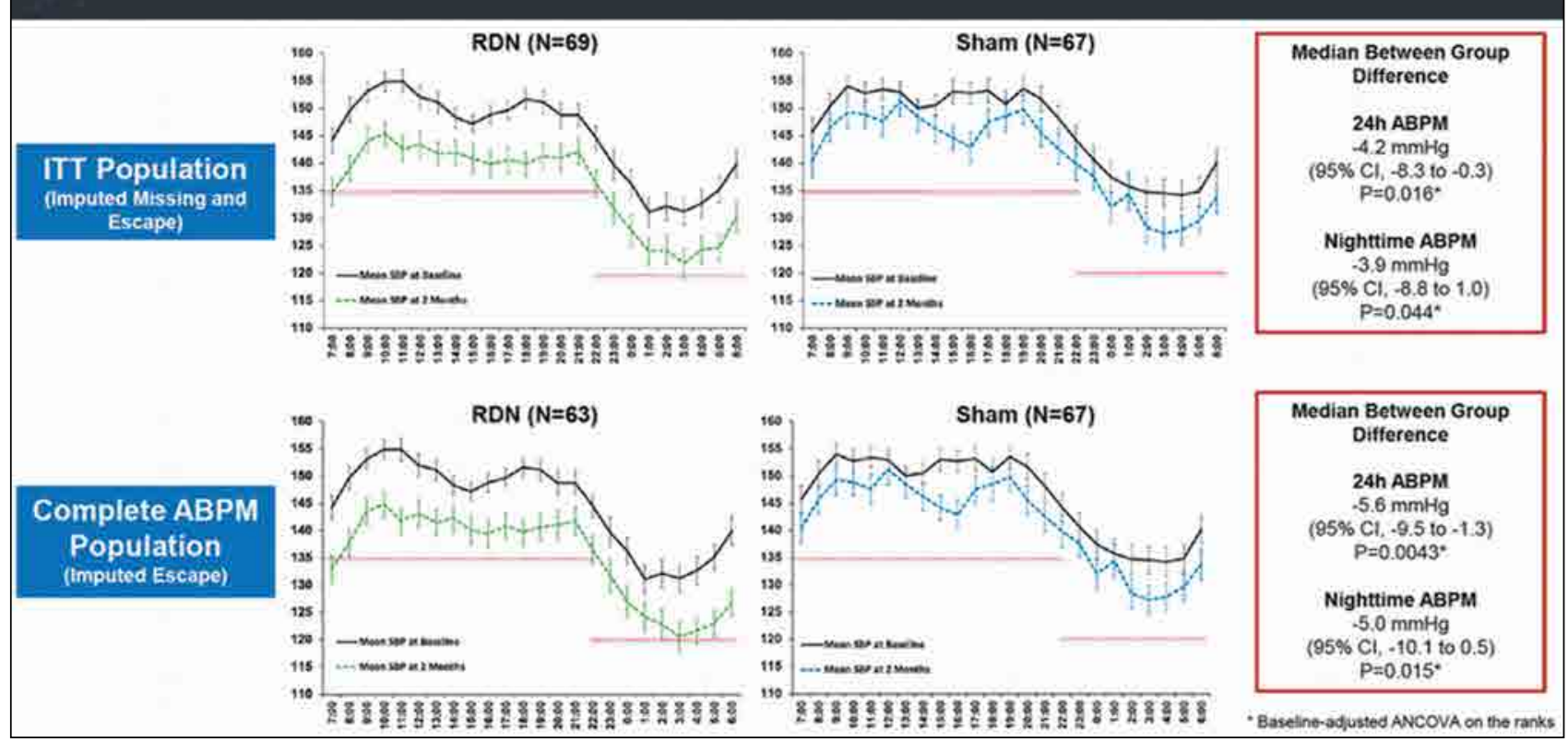

Apresentação durante o ACC 2021. ${ }^{18}$ 
Do ponto de vista individual, novamente, a educação do paciente e o apoio multiprofissional são necessários para se obter uma melhor eficácia anti-hipertensiva.

Para os casos de hipertensão comprovada já se dispõem de excelentes recursos farmacológicos que garantam o seu controle, mas naqueles que se mostram refratários começam a surgir recursos adicionais que poderão ser implantados no futuro próximo e dentre eles a expectativa maior é o da desnervação renal.

\section{Referências}

1. Introcaso L. História da medida da pressão arterial. HiperAtivo 1998;5:79-82.

2. Whelton P. Epidemiology of hypertension. Lancet 1994;344:101-6.

3. Malta DC GR, Machado IE, Freitas MIF, r Azeredo C, Szwarcwald CL. Prevalência da hipertensão arterial segundo diferentes critérios diagnósticos, Pesquisa Nacional de Saúde. Rev bras epidemiol 2018;21.

4. Vasan RS LM, Leip EP, Kannel WB, Levy D. Assessment of frequency of progression to hypertension in non-hypertensive participants in the Framingham Heart Study: a cohort study. Lancet 2001;358:1682-6.

5. Carey RM et al. Resistant hypertension: Detection, evaluation, and management a scientific statement from the American Heart Association. Hypertension 2018;72:e53-e90.

6. Mills KT BJ, Kelly TN, Reed JE, Kearney PM, Reynolds K, et al Global disparities of hypertension prevalence and control: a systematic analysis of population-based studies from 90 countries. Circulation 2016;134:441-450.

7. Carey RM MP, Bosworth HB, Whelton PK. Prevention and Control of Hypertension. JACC Health Promotion Series. J Am Coll Cardiol. 2018;71:2199-269.

8. Cook NR CJ, Hebert PR, Taylor JO, Hennekens CH. Implications of small reductions in diastolic blood pressure for primary prevention. Arch Intern Med 1995;155:701-9.

9. Souza IPMAd, Sampaio J, Vieira ML, Viana PA, Feitosa GF and Feitosa GS. The use of pedometer as a stimulus to the physical activity in the hypertensive patients with difficult-to-control hypertension: A controlled randomized study. Revista Científica Hospital Santa Izabel 2019;3:3-8.

10. Law M R MJK, Wald N J. BMJ 2009;2;338.

11. Bloom B. Continuation of initial antihypertensive medication after 1 year of therapy. Clin Ther 1998;20:671-81.

12. Calhoun D JD, Textor S, Goff DC, Murphy TP, Toto RG al. Resistant hypertension: Diagnosis, evaluation, and Treatment. A scientific statement from the American Heart Association Professional Education Committee of the Council for High Blood Pressure Research. Circulation 2008;117:e510-e526.

13. Daugherty SL PJ, Magid DJ, Tavel HM, Masoudi FA, Margolis KL et al. Incidence and prognosis of resistant hypertension in hypertensive patients. Circulation 2012;125:1635-1642.

14. Krieger EM DL, Giorgi DMA, Pereira AC, BarretoFilho JAS, Nogueira AR, et al. Spironolactone versus Clonidine as a fourth-drug therapy for resistant hypertension: The ReHOT randomized study (Resistant Hypertension Optimal Treatment). Hypertension 2018;71:681-90.

15. Esler MD KH, Sobotka PA, Schlaich MP, Schmieder RE, Bohm M. Renal sympathetic denervation in patients with treatment-resistant hypertension (the SYMPLICITY HTN-2 Trial): a randomised controlled trial. Lancet 2010;376.

16. Bhatt DL KD, O'Neill WW, D'Agostino R, Flack JM, Katzen BT et al. A controlled trial of renal denervation for resistant hypertension-SYMPLICITY HTN-3 N Engl J Med 2014;370:1393-1401.

17. Sardar P, Bhatt DL, Kirtane AJ et al. Sham-controlled randomized trials of catheter-based renal denervation in patients with hypertension. J Am Coll Cardiol 2019;73.

18. Azizi MKS, Saxena M, Gosse P, Reilly JP, Levy T et al. Ultrasound renal denervation for hypertension resistant to a triple medication pill (RADIANCE-HTN TRIO): A randomised, multicentre, single-blind, shamcontrolled trial. Lancet 2021;397:2476-2486.

19. Kandzari DB, Mahfoud F et al. Effect of renal denervation on blood pressure in the presence of antihypertensive drugs: 6-month efficacy and safety results from the SPYRAL HTN-ON MED proof-ofconcept randomised trial. Lancet 2018;391:2346-2355. 